\title{
EFFICIENT APPROACH FOR CONTENT BASED IMAGE RETRIEVAL USING MULTIPLE SVM IN YACBIR
}

\author{
Lakhdar LAIB $^{1}$ and Samy Ait-Aoudia ${ }^{2}$ \\ ${ }^{1,2}$ National High School of Computer Science ESI, Algiers, ALGERIA \\ 1_laibeesi.dz \\ s_ait_aoudia@esi.dz
}

\begin{abstract}
Due to the enormous increase in image database sizes, the need for an image search and indexing tool is crucial. Content-based image retrieval systems (CBIR) have become very popular for browsing, searching and retrieving images in different fields including web based searching, industry inspection, satellite images, medical diagnosis images, etc. The challenge, however, is in designing a system that returns a set of relevant images i.e. if the query image represents a horse then the first images returned from a large image dataset must return horse images as first responses. In this paper, we have combined YACBIR [7], a CBIR that relies on color, texture and points of interest and Multiple Support Vector Machines Ensemble to reduce the existing gap between high-level semantic and low-level descriptors and enhance the performance of retrieval by minimize the empirical classification error and maximize the geometric margin classifiers. The experimental results show that the method proposed reaches high recall and precision.
\end{abstract}

\section{KEYWORDS}

Content Based Image Retrieval, YACBIR, Feature extraction, Multiple Support Vector Machines, Classification

\section{INTRODUCTION}

With fast and exceptional growth in digital technology, a significant increase in the number of images produced by scientific, educational, medical, industrial, virtual museums, individual photograph collections and other applications raises several practical issues.

Content-based Image Retrieval (CBIR) system [1] [2] [3] [4] is the application of computer vision techniques which is used to extract similar images from an large image database. CBIR system is mainly used in various applications such as art collections, education and training, crime prevention, military field, architectural and engineering design, journalism and advertising, and web searches [5].

Simply stated, a CBIR system retrieves images corresponding to a query image by examining the image contents (low-level feature). Many image low-level features such as color, texture and shape are widely used [7]. The color aspect can be treated by techniques like averaging and histograms. The texture aspect can be achieved by using four descriptors: contrast, entropy, energy and inverse differential moment [8]. The shape aspect can be extracted by gradient or morphological operators [6] or by considering points of interest. [8].

Natarajan Meghanathan et al. (Eds) : ACSIT, SIPM, CMIT, CoNeCo - 2016

pp. 19-29, 2016. (C) CS \& IT-CSCP 2016

DOI : $10.5121 /$ csit.2016.60803 


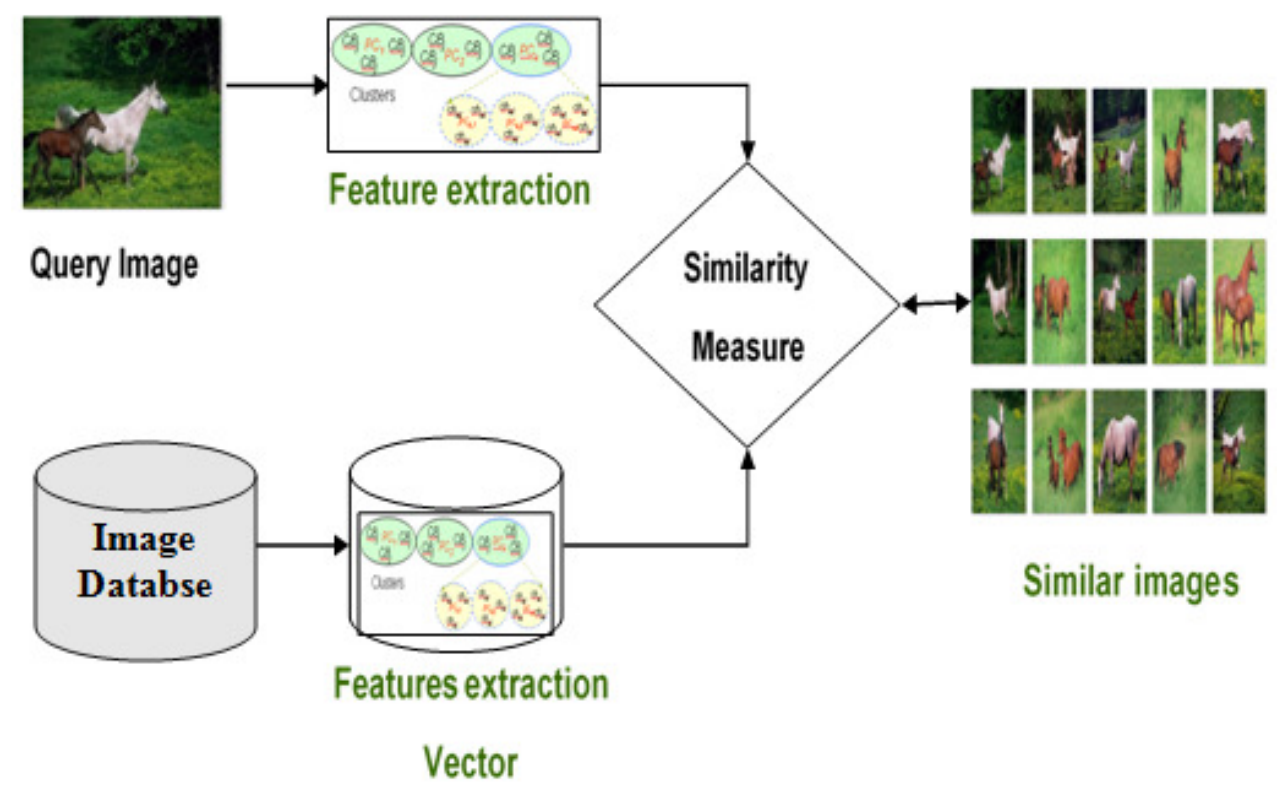

Figure 1: Content Based Image Retrieval Approach.

The main goal in CBIR system is searching the similar images from the database based on their content. To accomplish the retrieval task, CBIR system firstly computes and stores the feature vector for each image in the database. When the query image is introduced in the system, its feature vector will be extracted and compared to those of other images in the database. Then a series of similar images is returned to the user. Different similarity measures are used in the matching process [6].

This paper is organized as follows. We give in section 2 an overview of our proposed system. In section 3, concepts of SVM (Support Vector Machine) are reminded. Experimental results on sample datasets are given in section 4 . Section 5 gives conclusions.

\section{OVERVIEW OF PROPOSED SYSTEM}

\subsection{Method summary}

Step 1: Feature extraction $\mathrm{f}$ (Color, Texture, and Point of interest).

Step 2: Sum of weighted color, texture and points of interest features.

Step 3: Query image feature classification by Multiple Support Vector Machines (Find the class Label).

Step 4: Compute the distance between the Query image and images in the dataset (Class Label).

Step 5: Output Retrieved images (Similar images). 


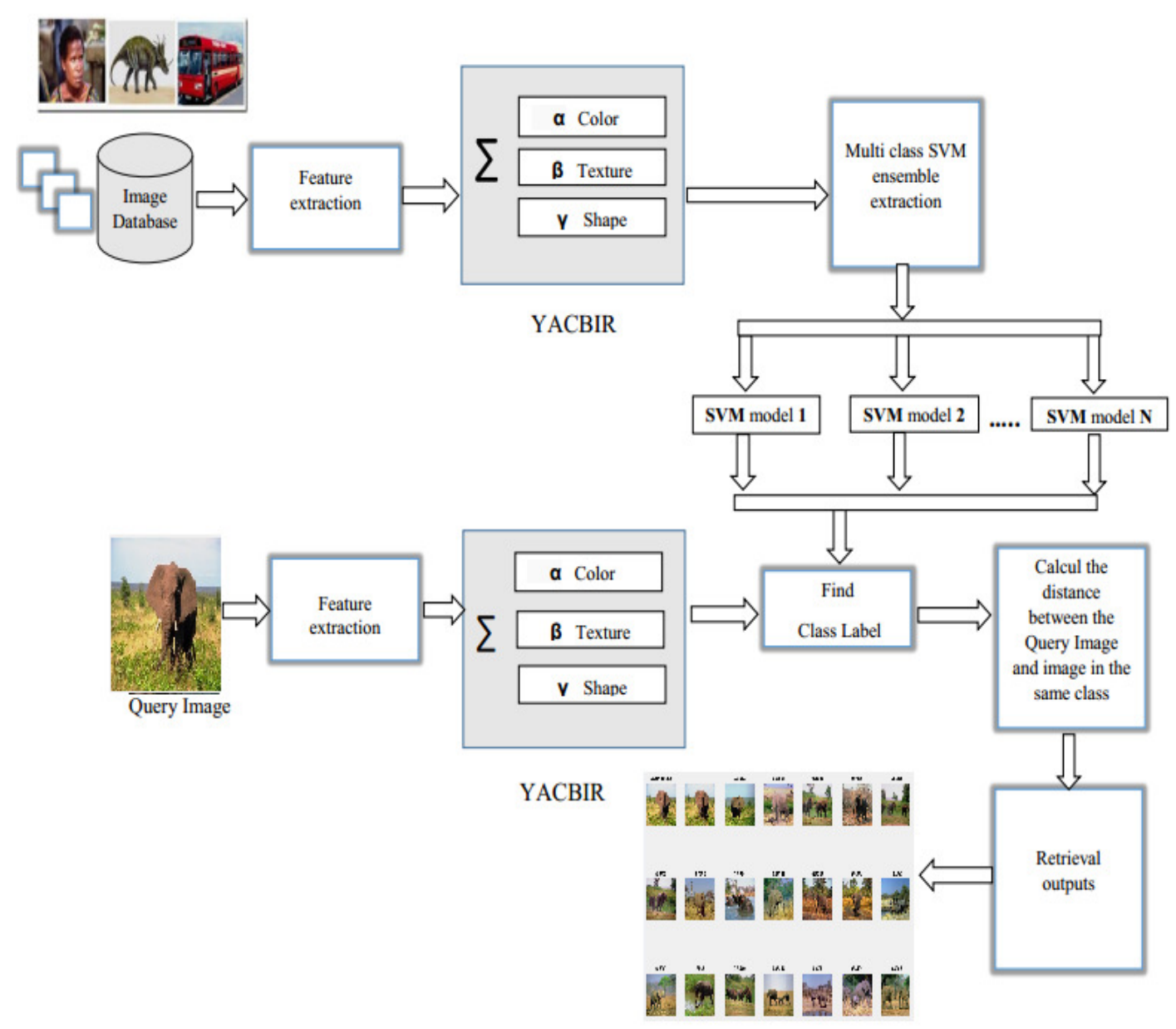

Figure 2: Overview of the process

\subsection{Feature extraction in YACBIR}

Feature extraction is a common way to improve the performance of the visual features and improve the efficiency of the CBIR systems. The best subset of features is selected by a heuristic search algorithm to improve the precision of CBIR system. Three types of features are used for image indexing [7].

Color - HSV

Texture -contrast, entropy, energy and inverse differential moment

Point of interest -Harris detector

The YACBIR system combines three characteristics of an image to compute a weighted similarity measure. The characteristics of the image are the color, texture and points of interest. Color and texture characteristics used are global while points of interest are local shape characteristics.

The similarity measure used in YACBIR is a sum of weighted color, texture and points of interest (shape) similarity measures. This similarity measure is given by:

$$
\mathrm{S}=\alpha . \mathrm{S} c+\beta . \mathrm{S} t+\gamma . \mathrm{S} s \quad \text { with }(\alpha+\beta+\gamma)=1
$$




\subsection{Support Vector Machines (SVM)}

In machine learning, support vector machines (SVM) are supervised learning models with learning algorithms. Given a set of data where each data is marked as belonging to one of two categories, a SVM training algorithm constructs a model which classifies new data in one of these two classes. Though SVMs are fundamentally developed for such binary classification case, they are extendable for multi-class problems [9].

\section{The SVM algorithm consists of two phases:}

\section{a. Training phase:}

Input learning data for a SVM classifier can consist of distance to border vectors, binary images, Zernike moments, and more. Each input data is represented by vector $\mathbf{x}_{i}$ with label $y_{i}= \pm 1,1 \leq i \leq l, l$ is the number of samples.

The decision boundary should classify all points correctly, thus

$$
y_{i}\left(\mathbf{w}^{\mathbf{T}} \mathbf{x}_{i}+b\right) \geq 1, \forall i
$$

The decision boundary can be found by solving the following constrained optimization problem:

Minimize $\frac{1}{2}\|\mathbf{w}\|^{2} \quad$ subjectto $y_{i}\left(\mathbf{w}^{\mathbf{T}} \mathbf{x}_{i}+b\right) \geq 1 \forall i$

The Lagrangian of this optimization problem is:

$$
L=\frac{1}{2}\|\mathbf{w}\|^{2}-\sum_{i} \alpha_{i}\left(y_{i}\left(\mathbf{w}^{\mathbf{T}} \mathbf{x}_{i}+b\right)-1\right) \alpha_{i} \geq 0 \forall i \mathrm{~T}
$$

the optimization problem can be rewritten in terms of $\alpha_{i}$ by setting the derivative of the Lagrangian to zero:

$$
\begin{aligned}
& \text { Maximize } W(\alpha)=\sum_{i} \alpha_{i}-\frac{1}{2} \sum_{i, j} \alpha_{i} \alpha_{j} y_{i} y_{j} \mathbf{x}_{i}^{T} \mathbf{x}_{j} \\
& \text { subjectto } \alpha_{i} \geq 0, \sum_{i} \alpha_{i} y_{i}=0 \forall i
\end{aligned}
$$




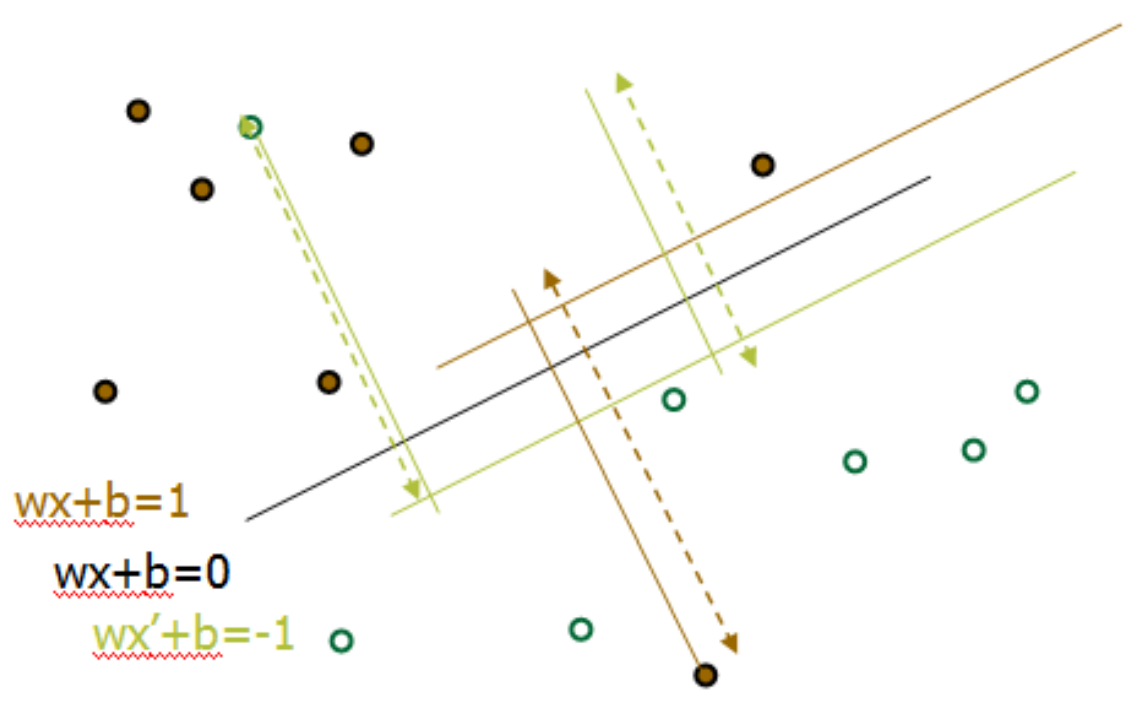

Figure 3: Support Vector Machine (SVM).

This quadratic programming problem is solved when:

$\alpha_{i}\left(y_{i}\left(\mathbf{w}^{\mathbf{T}} \mathbf{x}_{i}+b\right)-1\right)=0 \quad \forall i \mathbf{x}_{i}$ with $\alpha_{i}>0$

\section{b. Testing phase:}

The resulting classifier is applied to unlabelled images to decide whether they belong to the positive or the negative category. The label of $x$ is simply obtained by computing:

$\mathbf{w}=\sum_{j=1}^{s} \alpha_{t}{ }_{j} t_{j} \mathbf{x}_{t_{j}}$ with $t_{j}(j=1, \mathrm{~K}, s)$ the indices of the $s$ support vectors. Classify $\mathbf{z}$ as category 1 if the sum is positive, and category 2 otherwise.

\section{EXPERIMENTAL RESULTS}

The experiments in this section are performed on a workstation with an Intel Corei3 $2.10 \mathrm{GHz}$ CPU and 4 GB of RAM running Win 10.

We evaluate the effectiveness of the proposed method for Corel-1k which is a set of 1000 images that are subdivided into 10 sufficiently distinct semantic classes, including African people, seaside, bus, building, mountain, dinosaur, elephant, horses, flowers and food images some examples are depicted in Figure 4. 


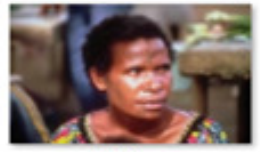

Africa

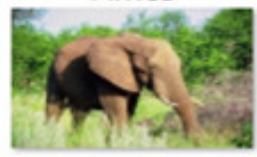

Elephants

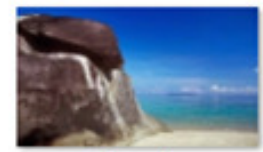

Beach

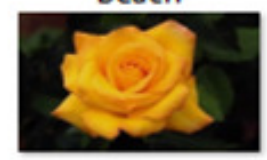

Flowers

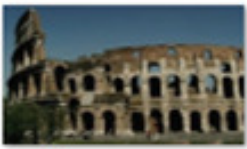

Monuments

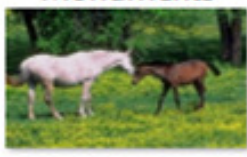

Horses



Buses

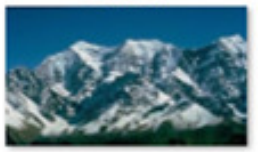

Mountains



Dinosaurs

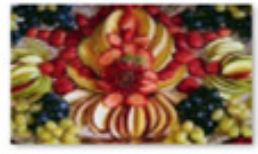

Food

Figure 4: Image database used (Corel-1k).

\section{2 Precision-recall}

Precision-versus-recall curve is the basic evaluation measure in information retrieval. They are defined as follow:

$$
\begin{aligned}
& \text { Precision }=\frac{\text { number of relevant images retrieved }}{\text { total number of relevant images retrieved }} \ldots . . . . \\
& \text { Recall }=\frac{\text { number of relevant images retrieved }}{\text { total number of relevant images in the database }}
\end{aligned}
$$

\begin{tabular}{|c|c|c|c|c|c|c|c|c|c|c|}
\hline Class & $\left|\begin{array}{l}\text { Lin et } \\
\text { a1.[10] }\end{array}\right|$ & $\begin{array}{l}\text { Elalami } \\
{[11]}\end{array}$ & \begin{tabular}{|l|} 
Poursist- \\
ani et al. \\
{$[12]$}
\end{tabular} & $\left|\begin{array}{l}\text { Guo et } \\
\text { a1.[13] }\end{array}\right|$ & \begin{tabular}{|l} 
Subra- \\
et al \\
{$[14]$}
\end{tabular} & $\begin{array}{c}\text { Walia } \\
\text { et al. } \\
{[15]}\end{array}$ & $\left|\begin{array}{l}\text { Irtaza et } \\
\text { al. [16] }\end{array}\right|$ & $\begin{array}{l}\text { Elalami } \\
{[17]}\end{array}$ & $\begin{array}{c}\text { Zeng et } \\
\text { a1.[18] }\end{array}$ & $\begin{array}{l}\text { Proposed } \\
\text { Method }\end{array}$ \\
\hline A frican & 68.30 & 70.30 & 70.20 & 84.70 & 69.75 & 51.00 & 65.00 & 72.60 & 2.50 & 68.40 \\
\hline Beach & 54.00 & 56.10 & 44.40 & 45.40 & 54.25 & 90.00 & 60.00 & 59.30 & 65.20 & 51.30 \\
\hline Monuments & 56.15 & 57.10 & 70.80 & 67.80 & 63.95 & 58.00 & 62.00 & 58.70 & 70.60 & 72.20 \\
\hline Buses & 88.80 & 87.60 & 76.30 & 85.30 & 89.65 & 78.00 & 85.00 & 89.10 & 89.20 & 85.50 \\
\hline Dinosal & 99.25 & 98.70 & 100.00 & 99.30 & 98.70 & 100.00 & 93.00 & 99.30 & 100.00 & 95.00 \\
\hline Elephants & 65.80 & 67.50 & 63.80 & 71.10 & 48.80 & 84.00 & 65.00 & 70.20 & 70.50 & 77.90 \\
\hline Flowe & 89.10 & 91.40 & 92.40 & 93.30 & 92.30 & 100.00 & 94.00 & 92.80 & 4.80 & 91.20 \\
\hline Horses & 80.25 & 83.40 & 94.70 & 95.80 & 89.45 & 100.00 & 77.00 & 85.60 & 91.80 & 85.50 \\
\hline Sount & 52.15 & 53.60 & 56.20 & 49.80 & 47.30 & 84.00 & 73.00 & 56.20 & 72.25 & 68.40 \\
\hline food & 73.25 & 74.10 & 74.50 & 80.80 & 70.90 & 38.00 & 81.00 & 77.20 & 78.80 & 77.90 \\
\hline verag & 72.70 & 73.90 & 74.39 & 77.30 & 72.51 & 78.30 & 75.50 & 76.10 & 80.57 & 77.33 \\
\hline
\end{tabular}

Table 1: Comparison precision proposed method with other methods 


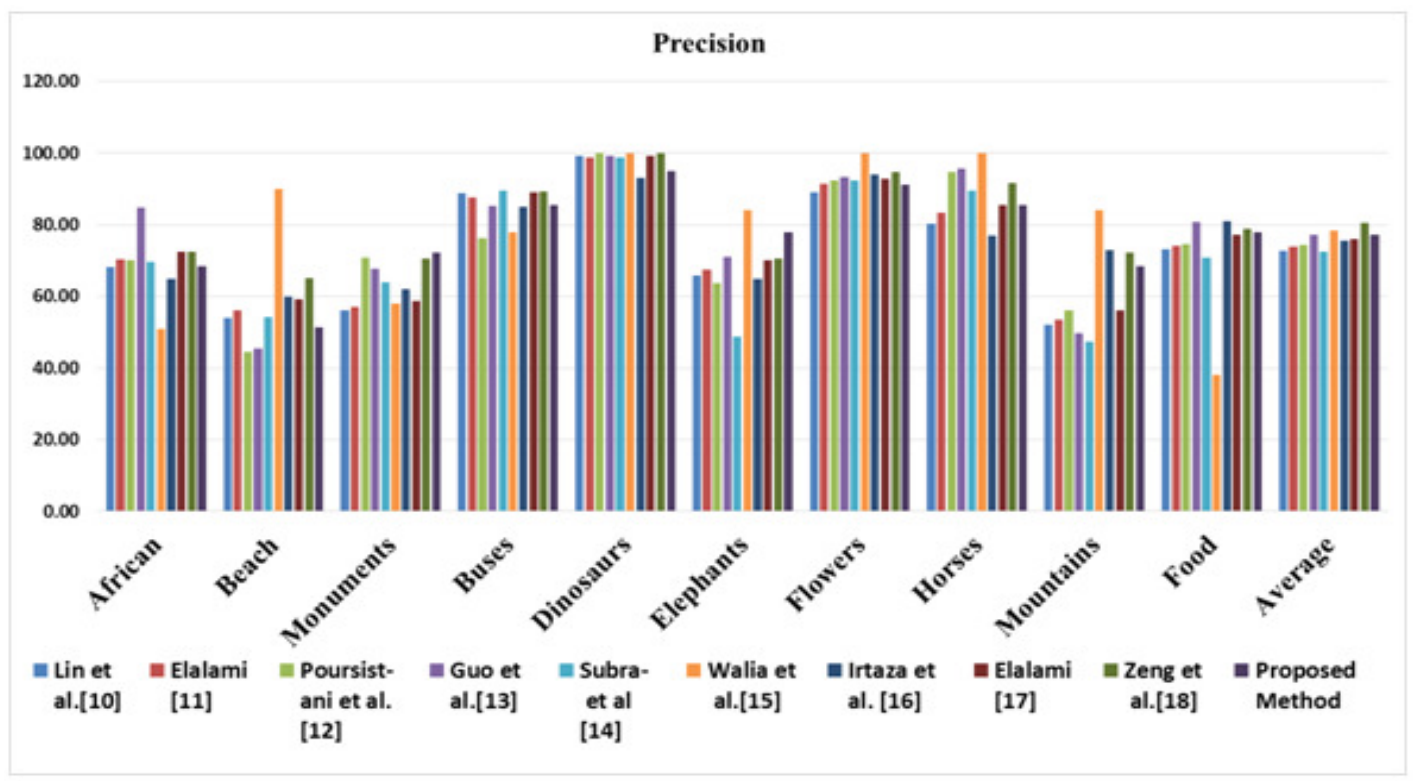

Figure 5. Precision of proposed approach compared to other methods for different semantic classes in the Corel database.

Table 2: Comparison recall proposed method with other methods

\begin{tabular}{|l|r|r|r|r|r|r|r|r|r|r|}
\hline & $\begin{array}{l}\text { Lin et } \\
\text { al.[10] }\end{array}$ & $\begin{array}{l}\text { Elalami } \\
{[11]}\end{array}$ & $\begin{array}{l}\text { Poursist- } \\
\text { ani et al. } \\
{[12]}\end{array}$ & $\begin{array}{l}\text { Guo et } \\
\text { al.[13] }\end{array}$ & $\begin{array}{l}\text { Subra- } \\
\text { et al } \\
{[14]}\end{array}$ & $\begin{array}{l}\text { Walia } \\
\text { et al. } \\
{[15]}\end{array}$ & $\begin{array}{l}\text { Irtaza et } \\
\text { al. [16] }\end{array}$ & $\begin{array}{l}\text { Elalami } \\
{[17]}\end{array}$ & $\begin{array}{l}\text { Zeng et } \\
\text { al.[18] }\end{array}$ & $\begin{array}{l}\text { Proposed } \\
\text { Method }\end{array}$ \\
\hline African & 14.10 & 15.30 & 14.04 & 16.94 & 13.95 & 10.20 & 13.00 & 16.10 & 14.50 & 14.46 \\
\hline Beach & 19.20 & 19.80 & 8.88 & 9.08 & 10.85 & 18.00 & 12.00 & 20.30 & 13.04 & 10.98 \\
\hline Monuments & 17.40 & 18.20 & 14.16 & 13.56 & 12.79 & 11.60 & 12.40 & 19.10 & 14.12 & 14.79 \\
\hline Buses & 12.10 & 11.60 & 15.26 & 17.06 & 17.93 & 15.60 & 17.00 & 12.60 & 17.84 & 18.07 \\
\hline Dinosaurs & 10.10 & 9.80 & 20.00 & 19.86 & 19.74 & 20.00 & 18.60 & 10.90 & 20.00 & 19.08 \\
\hline Elephants & 14.90 & 15.60 & 12.76 & 14.22 & 9.76 & 16.80 & 13.00 & 16.30 & 14.10 & 16.60 \\
\hline Flowers & 11.20 & 11.80 & 18.48 & 18.66 & 18.46 & 20.00 & 18.80 & 12.90 & 18.96 & 19.28 \\
\hline Horses & 13.40 & 13.90 & 18.94 & 19.16 & 17.89 & 20.00 & 15.40 & 14.40 & 18.36 & 18.37 \\
\hline Mountains & 21.30 & 22.80 & 11.24 & 9.96 & 9.46 & 16.80 & 14.60 & 23.60 & 14.45 & 14.34 \\
\hline Food & 13.20 & 13.80 & 14.90 & 16.16 & 14.18 & 7.60 & 16.20 & 14.80 & 15.76 & 16.73 \\
\hline Average & 14.68 & 15.20 & 14.86 & 15.46 & 14.50 & 15.66 & 15.10 & 16.10 & 16.11 & 16.27 \\
\hline
\end{tabular}






Figure 6. Precision of Proposed Approach Compared to Other Methods for Different Semantic Classes in the Corel Database
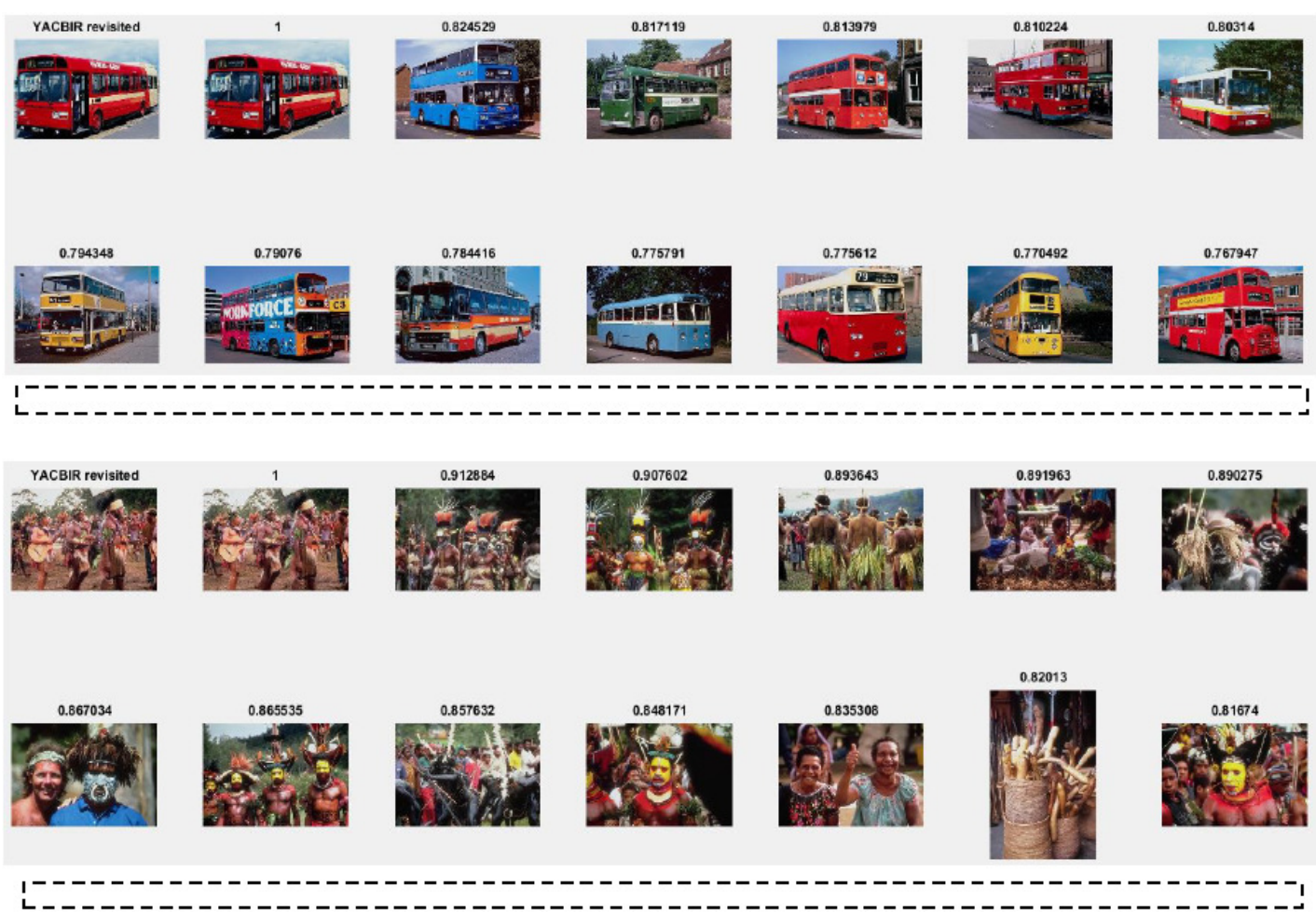


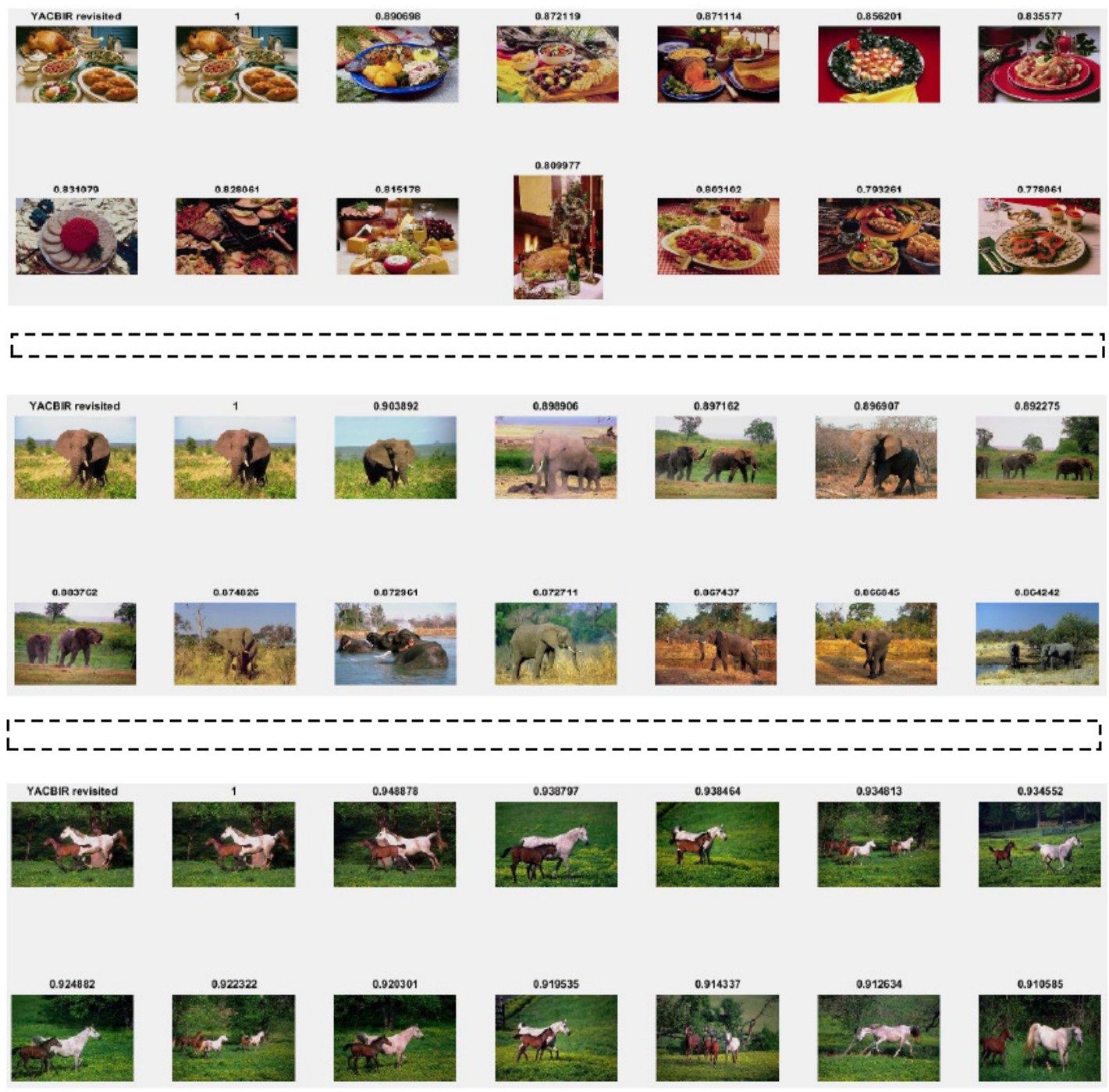

\section{CONCLuSiON}

In this paper, we proposed an efficient learning approach based on multiple SVMs (Support Vector Machines) and YACBIR system. From the test results, we show that retrieval performance of the proposed algorithm has quite good result in the image retrieval system. In future research, other classification techniques can be tested. Artificial Neural Networks (ANN) transfer functions and multiple SVMs kernel can be used to improve the classifier performance.

\section{REFERENCES}

[1] Query by image and video content: The QBIC system" S. W. Teng and G. Lu, "Codebook Generation in Vector Quantization Used for Image Retrieval," International Symposium on Intelligent Multimedia and Distance Education, 1999.

[2] Y. Liu, D. Zhang, G. Lu, and W. Ma, "A Survey of Content-based Image Retrieval with High-level Semantics," Pattern Recognition, Vol. 40, No. 1, 2007, pp. 262-282. 
[3] Datta, Ritendra, et al. "Image retrieval: Ideas, influences, and trends of the new age." ACM Computing Surveys (CSUR) 40.2 (2008): 5.

[4] Lew, Michael S., et al. "Content-based multimedia information retrieval: State of the art and challenges." ACM Transactions on Multimedia Computing, Communications, and Applications (TOMM) 2.1 (2006): 1-19.

[5] Ms. K.Arthi, Mr. J. Vijayaraghavan. (2013)"Content Based Image Retrieval Algorithm Using Colour Models", Chennai.

[6] Meenakshi Madhugunki, Dr. D.S.Bormane. (2011)"Comparsion of Different CBIR Techniques”, Pune.

[7] Ait-Aoudia, Samy, Ramdane Mahiou, and Billel Benzaid. "YACBIR: yet another content based image retrieval system." Information Visualisation (IV), 2010 14th International Conference. IEEE, 2010. Esmat

[8] Rashedi, Esmat, and Hossein Nezamabadi-pour. "Improving the precision of CBIR systems by feature selection using binary gravitational search algorithm." Artificial Intelligence and Signal Processing (AISP), 2012 16th CSI International Symposium on. IEEE, 2012.

[9] M. Mr.A.H.Karode and M. W.Pawade, "Texture image classification using support vector machine." International Journel of Computing Technology and Applications, 2012.

[10] C.H. Lin, R.T. Chen, Y.K. Chan, A smart content-based image retrieval system based on color and texture feature, Image Vis. Comput. 27 (6) (2009) 658-665.

[11] M.E. ElAlami, A novel image retrieval model based on the most relevant features, Knowl.-Based Syst. 24 (2011) 23-32.

[12] P. Poursistani, H. Nezamabadi-pour, R.A. Moghadam, M. Saeed, Image indexing and retrieval in JPEG compressed domain based on vector quantization, Math.Comput. Model. 57 (5-6) (2013) 10051017.

[13] J.M. Guo, H. Prasetyo, H.S. Su, Image indexing using the color and bit pattern feature fusion, J. Vis. Commun. Image R 24 (2013) 1360-1379.

[14] M. Subrahmanyam, Q.M.J. Wu, R.P. Maheshwari, R. Balasubramanian, Modified color motif cooccurrence matrix for image indexing and retrieval, Comput. M. Saeed, Image indexing and retrieval in JPEG compressed domain based on vector quantization, Math.A. Irtaza, M.A. Jaffar, E. Aleisa, T.S. Choi, Embedding neural networks for semantic association in content based image retrieval, Multimed. Tool Appl. 72 (2) (2014) 1911-1931.

[15] E. Walia, A. Pal, Fusion framework for effective color image retrieval, J. Vis.Commun. Image R 25 (2014) 1335-1348.

[16] A. Irtaza, M.A. Jaffar, E. Aleisa, T.S. Choi, Embedding neural networks for semantic association in content based image retrieval, Multimed. Tool Appl.72 (2) (2014) 1911-1931.Image retrieval using spatiograms of colors quantized by Gaussian Mixture Models. Neurocomputing Volume 171, 1 January 2016, Pages 673-684 72 (2) (2014) 1911-1931.

[17] M.E. ElAlami, A new matching strategy for content based image retrieval system, Appl. Soft Comput. 14 (2014) 407-418.

[18] S. Zeng, R. Huang, H. Wang, Z. Kang Image retrieval using spatiograms of colors quantized by Gaussian Mixture Models. Neurocomputing Volume 171, 1 January 2016, Pages 673-684 
[19] K. Mikolajczyk and C. Schmid, Scale and affi ne invariant interest point detectors, Int. J. Comput. Vis. 1 (2004), $63-86$.

[20] T. P. Minka and R. W. Picard, Interactive learning using a society of models, Pattern Recogn. 30 (1997), $565-581$.

\section{AUTHORS}

Lakhdar LAIB received the B Eng and MSc degrees in computer science from École nationale Supérieure en Informatique (Algeria) in 2010 and 2013, respectively. Since 2014, he has been pursuing PhD studies at École nationale Supérieure en Informatique (Algeria) under the supervision of Professor Samy Ait-Aoudia His primary research interests include statistical models and application to image segmentation, computer vision and pattern recognition.

Samy Ait- Aoudia received a DEA "Diplôme d'Etudes Approfondies" in image processing from Saint-Etienne University, France, in 1990. He holds a Ph.D. degree in computer science from the Ecole des Mines, Saint-Etienne, France, in 1994. He is currently a Professor of computer science at the National High School in Computer Science at Algiers/Algeria, where he is involved in teaching BSc and MSc levels in computer science and software engineering. His areas of research include image processing, CAD/CAM and constraints management in solid modeling.
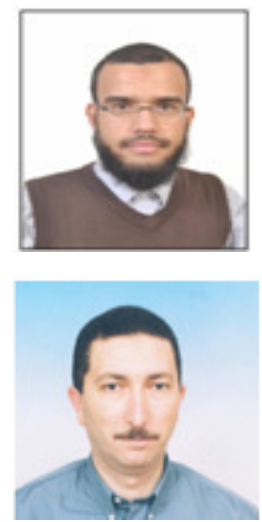